International Journal of Modern Physics A

(C) World Scientific Publishing Company

\title{
TWO PARTICLE STATES IN A BOX AND THE $S$-MATRIX IN MULTI-CHANNEL SCATTERING
}

\author{
CHUAN LIU, XU FENG and SONG HE \\ School of Physics, Peking University \\ Beijing 100871, China
}

\begin{abstract}
Using a quantum mechanical model, the exact energy eigenstates for two-particle twochannel scattering are studied in a cubic box with periodic boundary conditions. A relation between the exact energy eigenvalue in the box and the two-channel $S$-matrix elements in the continuum is obtained. This result can be viewed as a generalization of the well-known Lüscher's formula which establishes a similar relation in elastic scattering.

Keywords: lattice QCD; hadron scattering; finite volume technique.
\end{abstract}

\section{Introduction}

Scattering experiments play an important role in the study of interactions among particles. In the case of low-energy hadron-hadron scattering, experimental results on the phase shifts are available 1. On the theoretical side, low-energy hadronhadron scattering can be studied with a non-perturbative method, like Lattice QCD. In a typical lattice calculation, energy eigenvalues of two-particle states with definite symmetry are obtained. Therefore, it is important to relate the energy eigenvalues which are available through lattice calculations to the scattering phases which are obtained in the scattering experiments. This was accomplished in a series of papers by Lüscher ${ }^{2}$ for a cubic box topology. This formula, now known as Lüscher's formula, has been utilized in a number of applications, e.g. linear sigma model in the broken phase 3 , and also in quenched 4 and unquenched ${ }^{[5]} \mathrm{QCD}$.

For hadron scattering at low energies, elastic scattering dominates. However, when the energy of the scattering process exceeds some threshold, inelastic scattering starts to contribute. In the case of pion-pion scattering, for example, the scattering process is elastic below the four pion and the two kaon threshold. Although the four pion threshold is in fact below the two kaon threshold, four pion final states will not contribute significantly due to its weak chiral coupling to the two pion initial state. Experimental investigations also supports this argument. It is then interesting to study the relation between the multi-channel two-particle states and the scattering phases in general. In this work, we report a relation between the energy of a two particle state in a finite cubic box and the scattering matrix parameters. Further details are provided in Ref. $\underline{6}$. 


\section{The Quantum Mechanical Model of Two Channel Scattering}

We study a non-relativistic quantum mechanical model of two-channel scattering with the following Hamiltonian:

$$
H=\left(\begin{array}{cc}
-\frac{1}{2 m_{1}} \nabla^{2} & 0 \\
0 & E_{T}-\frac{1}{2 m_{2}} \nabla^{2}
\end{array}\right)+\left(\begin{array}{cc}
V_{1}(r) & \Delta(r) \\
\Delta^{*}(r) & V_{2}(r)
\end{array}\right) .
$$

with the potential vanishes for $r>R . E_{T}>0$ designates a positive threshold energy of the second channel.

Energy eigenstates of the Hamiltonian (11) with energy $E$ can be decomposed into spherical harmonics: $\Psi_{i}(\mathbf{r})=\sum_{l, m} Y_{l m}(\hat{\mathbf{r}}) \psi_{i ; l m}(r)$ where the radial wave-functions $\psi_{i ; l m}(r)$ with $i=1,2$ satisfy the usual radial Schrödinger equation. Concerning the coupled radial differential equations, the following statement can be proven 6 . If the matrix valued potential $V(r)$ is such that every matrix element of $r^{2} V(r)$ is analytic around $r=0$ and that $\lim _{r \rightarrow 0} r^{2} V(r)=0$, then the coupled radial differential equations has two finite, linearly independent solutions near $r=0: u_{l ; j}^{(i)}(r)$, with $i=1,2$, $j=1,2$ such that: $u_{l ; j}^{(i)}(r) \sim r^{l} \delta_{i j}$.

At large $r$ where the potential $V(r)$ vanishes, the wave function of the scattering state can be chosen to have particular forms:

$$
\Psi^{(1)}(\mathbf{r}) \stackrel{r \rightarrow \infty}{\longrightarrow}\left(\begin{array}{c}
e^{i \mathbf{k}_{1} \cdot \mathbf{r}}+f_{11}\left(\hat{\mathbf{k}}_{1} \cdot \hat{\mathbf{r}}\right) \frac{e^{i k_{1} r}}{r} \\
f_{21}\left(\hat{\mathbf{k}}_{1} \cdot \hat{\mathbf{r}}\right) \sqrt{\frac{m_{2}}{m_{1}}} \frac{e^{i k_{2} r}}{r}
\end{array}\right) .
$$

This wave function has the property that in the remote past, it becomes an incident plane wave in the first channel with definite wave vector $\mathbf{k}_{1}$. It is an eigenstate of the full Hamiltonian with energy: $E=\mathbf{k}_{1}^{2} /\left(2 m_{1}\right)$. Similarly, if the energy $E>E_{T}$, one can also build another eigenstate of the Hamiltonian $\Psi^{(2)}(\mathbf{r})$, which in past becomes an incident wave in the second channel with energy $E=E_{T}+\mathbf{k}_{2}^{2} /\left(2 m_{2}\right)$.

In partial wave analysis, one decomposes the coefficients: $f_{i j}$ (scattering applitudes) into spherical harmonics. The scattering eigenstate in Eq. (2) can aslo be decomposed accordingly with the radial wave-functions:

$$
w_{l}^{(1)}(r) \simeq\left(\begin{array}{c}
\frac{1}{2 i k_{1} r}\left[S_{11}^{(l)} e^{i k_{1} r}+(-)^{l+1} e^{-i k_{1} r}\right] \\
\frac{1}{2 i \sqrt{k_{1} k_{2} r}} \sqrt{\frac{m_{2}}{m_{1}}} S_{21}^{(l)} e^{i k_{2} r}
\end{array}\right),
$$

and a similar equation for $\Psi^{(2)}(\mathbf{r})$ and $w_{l}^{(2)}(r)$. It is obvious that the two radial wave functions $w_{l}^{(1)}(r)$ and $w_{l}^{(2)}(r)$ thus defined form a linearly independent basis. They are hence linear superpositions of the general solutions: $u_{l}^{(1)}(r)$ and $u_{l}^{(2)}(r)$. The converse is of course also true.

An important physical property is that the matrix elements which enter the expansion, namely $S_{i j}^{(l)}$, form a $2 \times 2$ unitary matrix. In practice, this two-channel $S$-matrix is usually parameterized as:

$$
S^{(l)}(E)=\left(\begin{array}{cc}
\eta_{l} e^{2 i \delta_{1}^{l}} & i \sqrt{1-\eta_{l}^{2}} e^{i\left(\delta_{1}^{l}+\delta_{2}^{l}\right)} \\
i \sqrt{1-\eta_{l}^{2}} e^{i\left(\delta_{1}^{l}+\delta_{2}^{l}\right)} & \eta_{l} e^{2 i \delta_{2}^{l}}
\end{array}\right),
$$


where the parameters: $\delta_{1}^{l}, \delta_{2}^{l}$ and $\eta_{l}$ are all real functions of the energy $E$.

\section{Energy Eigenfunctions on a Torus}

When enclosed in a cubic, periodic box with finite extension $L$, the Schrödinger equation of the system takes a similar form except that the potential is periodically extended and the eigenfunction has to satisfy the periodic boundary condition: $\left[\mathrm{H}_{0}+\right.$ $\left.V_{L}(\mathbf{r})\right] \psi(\mathbf{r})=E \psi(\mathbf{r}), \quad \psi(\mathbf{r}+L \mathbf{n})=\psi(\mathbf{r})$. where $H_{0}$ is the free Hamiltonian and the periodically extended potential is: $V_{L}(\mathbf{r}) \equiv \sum_{\mathbf{n}} V(\mathbf{r}+L \mathbf{n})$. The eigenvalue of the Hamiltonian now becomes discrete with smooth eigenfunctions. It is also convenient to partition the whole space into two regions. In the inner region, every point satisfies the condition: $|\mathbf{r}|<R, \bmod (L)$. In the outer region: $\Omega=\{\mathbf{r}|:| \mathbf{r} \mid>R, \bmod (L)\}$, where the interaction potential $V_{L}(\mathbf{r})=0$ and the Schrödinger equation of the system reduces to two decoupled Helmholtz equations: $\left(\nabla^{2}+k_{i}^{2}\right) \psi_{i}(\mathbf{r})=0, i=1,2$.

It it easy to see that: $\Psi(\mathbf{r} ; E)=\sum_{l m}\left[\sum_{i=1}^{2} b_{l m}^{(i)} u_{l}^{(i)}(r)\right] Y_{l m}(\mathbf{n})$ solves the

Schrödinger equation in the inner region for $|\mathbf{r}|<R$ with $b_{l m}^{(j)}$ being non-vanishing coefficients. In the outer region $\Omega$, the solution must be linear superposition of the singular periodic solutions of Helmholtz equation $[6$, we thus obtain a set of homogeneous linear equations. In order to have non-trivial solutions for the coefficients, the corresponding matrix has to be singular. This condition then gives:

$$
\left|\begin{array}{cc}
U_{l^{\prime} m^{\prime} ; l m}^{(1)}-S_{11}^{(l)} \delta_{l^{\prime} l} \delta_{m^{\prime} m} & \sqrt{\frac{k_{2} m_{2}}{k_{1} m_{1}}} S_{21}^{(l)} \delta_{l^{\prime} l} \delta_{m^{\prime} m} \\
\sqrt{\frac{k_{1} m_{1}}{k_{2} m_{2}}} S_{12}^{(l)} \delta_{l^{\prime} l} \delta_{m^{\prime} m} & U_{l^{\prime} m^{\prime} ; m}^{(2)}-S_{22}^{(l)} \delta_{l^{\prime} l} \delta_{m^{\prime} m}
\end{array}\right|=0,
$$

where the unitary matrices $U^{(i)}$ are specific functions of the energy 6 .

In most lattice calculations, the symmetry sector that is easiest to investigate is the invariant sector: $A_{1}^{+}$. We therefore focus on this particular symmetry sector. In the first order approximation, if we neglect the mixing between the $s$-wave and $g$-wave, we have for the $A_{1}^{+}$sector:

$$
\cos \left(\Delta_{1}+\Delta_{2}-\delta_{1}^{0}-\delta_{2}^{0}\right)=\eta_{0} \cos \left(\Delta_{1}-\Delta_{2}-\delta_{1}^{0}+\delta_{2}^{0}\right) .
$$

where we have also used the special parametrization (4) for the $s$-wave $S$-matrix elements. The quantities appearing above, namely $\delta_{1}^{0}, \delta_{2}^{0}, \eta_{0}, \Delta_{1}$ and $\Delta_{2}$, are all functions of the energy: $E=k_{1}^{2} /\left(2 m_{1}\right)=E_{T}+k_{2}^{2} /\left(2 m_{2}\right)$. Eq. (6) is the simplified formula for the $s$-wave $S$-matrix elements when contaminations from higher angular momentum (mainly from $l=4$ ) are neglected. This relation is helpful since it provides a constraint on the four physical quantities. If the mixing of the $g$-wave is taken into account, the corresponding formula becomes more complicated $[$ ] Although we worked out the formulae in a cubic box, similar relations can also be obtained for general rectangular box following the strategies outlined in Ref. 7 .

Finally, let us speculate about possible extension to the case of massive field theory. As in the case of single channel scattering 28 , one can work in the Bethe- 
Salpeter formalism for the two particle states and the results reported here can possibly be generalized to field theory. Details for this are now under investigation.

\section{Conclusions}

In this work, we have studied two-particle two-channel scattering states in a cubic box with periodic boundary conditions. Assuming that energy eigenstates are only two-particle states, the relation of the exact energy eigenvalues in the box and the physical parameters in the coupled channel $S$-matrix elements in the continuum is found. This formula can be viewed as a generalization of the well-known Lüscher's formula to the coupled channel situation (inelastic scattering). In particular, we show that the two-channel $S$-matrix elements in the $s$-wave are related to the energy of the two-particle system by a simple identity, if contaminations from higher angular momentum sectors are neglected. This relation is non-perturbative in nature and it will help us to establish connections between the $S$-matrix parameters in the multi-channel scattering with the energy eigenvalues which are in principle accessible in lattice calculations.

\section{Acknowledgments}

We would like to thank H. Q. Zheng for helpful discussions.

\section{References}

1. P. Truöl, hep-ex/0012012, 2000; M.J. Matison et al., Phys. Rev. D 9, 1872 (1974); N.O. Johannesson and J.L. Petersen, Nucl. Phys. B 68, 397 (1973); A. Karabouraris and G. Shaw, J. Phys. G 6583 (1980); A.D. Martin, Nucl. Phys. B 179, 33 (1981).

2. M. Lüscher, Commun. Math. Phys. 105, 153 (1986); M. Lüscher and U. Wolff, Nucl. Phys. B 339, 222 (1990); M. Lüscher, Nucl. Phys. B 354, 531 (1991); M. Lüscher, Nucl. Phys. B 364, 237 (1991).

3. M. Goeckeler, H.A. Kastrup, J. Westphalen, and F. Zimmermann, Nucl. Phys. B 425, 413 (1994).

4. R. Gupta, A. Patel, and S. Sharpe, Phys. Rev. D 48, 388 (1993); M. Fukugita, Y. Kuramashi, H. Mino, M. Okawa, and A. Ukawa, Phys. Rev. D 52, 3003 (1995); S. Aoki et al., Nucl. Phys. (Proc. Suppl.) B 83, 241 (2000); S. Aoki et al., Phys. Rev. D 66, 077501 (2002); C. Liu, J. Zhang, Y. Chen, and J.P. Ma, Nucl. Phys. B 624, 360 (2002); S. Aoki et al., Phys. Rev. D 67014502 (2003).

5. T. Yamazaki et al., Phys. Rev. D 70074513 (2004).

6. S. He, X. Feng and C. Liu JHEP 0507, 011 (2005).

7. X. Li and C. Liu, Phys. Lett. B 587100 (2004); X. Feng, X. Li, and C. Liu, Phys. Rev. D 70014505 (2004).

8. C.h. Kim, C.T. Sachrajda and S. Sharpe, hep-lat/0507006 2005. 